\title{
Misinterpretation of allergic bronchopulmonary aspergillosis/ allergic bronchopulmonary mycosis due to diverse characteristics in different clinical stages
}

\author{
Haijun Feng ${ }^{1 \#}$, Ping Lv $^{2 \#}$, Xiaoxia Ren ${ }^{3}$, Huaping Dai ${ }^{3}$, Ting Yang ${ }^{3}$ \\ ${ }^{1}$ Department of Respiratory Medicine, Beijing First Hospital of Integrated Chinese and Western Medicine, Beijing 100026, China; ${ }^{2}$ Department of \\ Internal Medicine, Beijing Chaoyang District Health Service Center, Beijing 100013, China; ${ }^{3}$ Department of Pulmonary and Critical Care Medicine, \\ Center of Respiratory Medicine, China-Japan Friendship Hospital, National Clinical Research Center for Respiratory Disease, Beijing 100029, \\ China \\ Contributions: (I) Conception and design: H Feng, T Yang; (II) Administrative support: T Yang; (III) Provision of study materials or patients: H Feng, \\ P Lv; (IV) Collection and assembly of data: H Feng, P Lv, X Ren, H Dai; (V) Data analysis and interpretation: H Feng; (VI) Manuscript writing: All \\ authors; (VII) Final approval of manuscript: All authors. \\ \#These authors contributed equally to this work. \\ Correspondence to: Ting Yang, MD, PhD. Department of Pulmonary and Critical Care Medicine, Center of Respiratory Medicine, China-Japan \\ Friendship Hospital, National Clinical Research Center for Respiratory Disease, No. 2, Yinghuayuan East Street, Chaoyang District, Beijing 100029, \\ China. Email: dryangting@qq.com.
}

Background: Allergic bronchopulmonary mycosis (ABPM) is a complex pulmonary disorder caused by a hyperimmune response to the endobronchial growth of certain fungi. This study aims to aid physicians in better understanding the need for earlier recognition of ABPM.

Methods: Patients with a confirmed diagnosis of ABPM after evaluation were analyzed retrospectively. Clinical features, previous diagnoses and potential diagnostic errors, laboratory findings were reviewed and compiled.

Results: Fifty-seven patients were diagnosed with ABPM in which 22 were misdiagnosed. Eosinophilia was observed in 11 patients and an elevated total serum $\mathrm{Ig}$ E level greater than 1,000 kU/L was detected in 25 of the 57 patients. All of the patients were tested for specific Ig E in which positive to different fungi. The two most common abnormalities found on chest high-resolution computed tomography (HRCT) exams were central bronchiectasis and mucus plugs. A mild to moderate obstructive pattern of lung function was observed in 32 cases. By bronchofiberscopic observation, bronchial lumen was seen to have significant inflammation in 12 patients. After 3 weeks of treatment, a decreased serum total $\mathrm{Ig} \mathrm{E}$ value was observed.

Conclusions: This study highlights the importance of increasing awareness and understanding of nonAspergillus-ABPM among physicians and draws attention to the need for establishing more elaborate diagnostic criteria for non-Aspergillus-ABPM, which is a rare kind of disease.

Keywords: Allergic bronchopulmonary mycosis (ABPM); allergic bronchopulmonary aspergillosis (ABPA); diagnosis

Submitted Apr 27, 2019. Accepted for publication Sep 29, 2019.

doi: $10.21037 /$ jtd.2019.10.78

View this article at: http://dx.doi.org/10.21037/jtd.2019.10.78

(c) Journal of Thoracic Disease. All rights reserved. 


\section{Introduction}

Allergic bronchopulmonary mycosis (ABPM) is a pulmonary hypersensitivity-mediated disease, which was characterized by sensitization to environmental fungi, central bronchiectasis, peripheral and pulmonary eosinophilia, and recurrent fleeting radiographic infiltrate (1-3). This disease was first described as allergic bronchopulmonary aspergillosis (ABPA) by Hinson et al. in the United Kingdom who recognized it in asthmatics with uncontrolled asthma, peripheral blood eosinophilia, recurrent pulmonary infiltrates and sputum culture positive for Aspergillus fumigatus (4). Since then, a growing number of studies found that several different fungi have also been implicated in causing a syndrome similar to ABPA, including Aspergillus flavus (5), Aspergillus niger (6), Bipolaris spp (7), Candida albicans (8), Curvularia lunata, Drecbslera bawaiiensis (9) and Schizophyllum commune (10). The complex disease is an airway disorder, which is associated with two most important underlying conditions including asthma and cystic fibrosis in primarily patients $(11,12)$.

The ISHAM Working Group has proposed a latest clinical staging of ABPA, which has identified ABPA into seven clinical stages including stage 0 (diagnosed as ABPA but has no signs and symptoms), stage 1 (acute, 1a: with mucoid impaction, 1b: without mucoid impaction), stage 2 (response), stage 3 (exacerbation), stage 4 (remission), stage 5 (5a: treatment dependent ABPA, 5b: glucocorticoid dependent asthma), stage 6 (advanced ABPA). Several studies indicated that many patients with stage 1 , stage 2 , stage 3 and stage 4 can enter complete remission, while stage 5 and stage 6 may lead to lung fibrosis and irreversible lung damage (13). Therefore, early diagnosis and treatment is essential to lower the risk of advancement to stage 5 or stage 6, which could prevent long-term lung tissue damage (14). However, previous studied showed that the mean latency of diagnostic delay was as long as 10 years in patients with ABPM, which was correctly diagnosed at later stages eventually $(15,16)$. Thus, the early diagnosis of $\mathrm{ABPM}$ is an important clinical issue.

Hence, a call was made to alert medical practitioners to identify this disease entity of ABPM as early as possible, especially in the high-risk patients with asthma or cystic fibrosis. In this study, we reported a relatively large series of ABPM patients who have suffered long duration of this disease, and even some of whom were at the irreversible stage when the correct diagnosis was made finally. Further, the clinical information of these patients was retrospectively analyzed regarding clinical presentation, laboratory and radiological evaluations. This will improve the early diagnosis and outcomes for ABPM patients hopefully.

\section{Methods}

This was a single-center retrospective study from the department of respiratory and critical care medicine using the clinical information system. The data of all patients diagnosed as ABPM from Dec 2015 to Dec 2017 were collected. Patients were excluded if they met any of the following criteria: (I) a history of pulmonary surgery; (II) previous diagnosis of advanced cancer or other severe diseases; (III) pregnancy; (IV) any immunosuppressive diseases (including: chronic renal failure, uncontrolled diabetes mellitus, chronic liver failure and cytotoxic therapy) or intake of systemic corticosteroids within 4 weeks; (V) mental or cognitive disorder.

Rosenberg-Patterson (R-P)-criteria was used to diagnose ABPA, including major criteria and minor criteria. Major Criteria: (I) asthma; (II) presence of fleeting or fixed pulmonary opacities on chest radiograph; (III) immediate cutaneous hypersensitivity reaction to Aspergillus fumigatus; (IV) total serum Ig E elevated, more than 1,000 IU/mL; (V) precipitating antibodies against Aspergillus fumigatus; (VI) peripheral blood eosinophilia; (VII) central or proximal bronchiectasis with normal tapering of distal bronchi. Minor criteria: (I) golden brown sputum plugs in expectorant; (II) positive sputum culture for Aspergillus species; (III) late (arthus-type) skin reactivity to Aspergillus fumigatus. The diagnosis of ABPM was made by a multidisciplinary team (pulmonary physician, radiologist, microbiologist) when at least 6 of the primary diagnostic R-P criteria were met (17). Apart from detailed demographic data, clinical history and physical examination, all subjects underwent the following investigations to achieve a definite diagnosis of ABPM: peripheral blood eosinophil count, total serum Ig E levels, fungus-specific Ig E levels, sputum microscopy and culture for fungus, high-resolution computed tomography (HRCT) of the chest. Lung function test and bronchofiberscopy were also performed in some patients. The indications included recurrent cough and sputum with invasion in lungs (nontypical imaging of ABPA), and non-response to empirical anti-infective therapy.

\section{Total eosinophil count}

The total leucocyte count was initially determined using 
an auto-analyzer (LH-750; Beckman Coulter, Mumbai, India). The percentage of differential leucocyte count was measured by counting and classifying 100 white blood cells on a peripheral blood smear. The total eosinophil count was obtained by multiplying the percentage with the total leucocyte count.

\section{Serum total Ig E and fungus-specific Ig E levels}

The total Ig E levels were assessed using quantitative enzyme-linked immunosorbent assay (Demeditec diagnostics GmbH, Kiel, Germany), while the fungusspecific Ig E levels were assayed using fluorescent enzyme immunoassay (UniCap Systems; Phadia, Stockholm, Sweden).

\section{Sputum culture}

Sputum samples were examined (smear and culture) for any pathogenic fungus in 57 patients. All the sputum samples were treated with $\mathrm{N}$-acetyl-cysteine (NALC) and were examined for fungal hyphae using calcofluor dye with potassium hydroxide and cultured using Sabouraud glucose agar medium. Further, mycelial fungi were identified using a slide culture technique.

\section{HRCT of the chest}

HRCT was performed using a multiple-detector CT scanner (LightSpeed Plus; GE Medical Systems; Slough, UK) with a matrix size of $512 \times 512$. The diagnosis of bronchiectasis and high-attenuation mucus on HRCT chest was made using the previously described criteria (18).

\section{Pulmonary function test}

Pulmonary function test was performed on a dry rolling seal spirometer (Spiroflow; PK Morgan Ltd.; Kent, UK) to determine the lung function measurements as previously described (19). Age, gender, height and spirometry data were recorded for the patients.

\section{Bronchofiberscopy}

Bronchofiberscopy was performed within 2 weeks of presentation and after stabilization of symptoms. During bronchofiberscopy, bronchial washings were obtained and cultured for growth of fungi. Further, $50 \mathrm{~mL}$ aliquots of sterile $0.9 \%$ saline were infused into the right middle lobe or lingula, gently suctioned, and then processed for cell counts and differentials. The mucosal specimens were also obtained by bronchofiberscopy, and then fixed in $10 \%$ formalin and routinely processed for staining with hematoxylin and eosin (HE). In addition, bronchofiberscopy was not performed in those patients, who met Rosenberg's criteria, had expectoration of mucus plugs, or rejected bronchofiberscopy due to concern of exacerbation of asthmatic symptoms.

\section{Treatment}

The patients were treated with glucocorticoids, itraconazole or glucocorticoid and itraconazole. The glucocorticoid was used as the following regimen: prednisolone $0.5 \mathrm{mg} / \mathrm{kg}$ for 4 weeks, $0.25 \mathrm{mg} / \mathrm{kg}$ for 4 weeks, $0.125 \mathrm{mg} / \mathrm{kg}$ for 4 weeks; then tapered by $5 \mathrm{mg}$ every week to continue for a total duration of at least 4 months. Antifungal agent itraconazole was used as a dose of $200 \mathrm{mg}$ twice a day for 4 months. The patients were followed up with history and physical examination, chest radiograph, serum total Ig E level and the number of eosinophil count every 4 weeks.

\section{Statistical analysis}

The data were presented in a descriptive fashion as number (percentage), or median (interquartile range), as required. The differences between continuous variables were analyzed using Kruskal-Wallis test, while the categorical variables were compared using Chi-square test. Differences with a P value of lower than or equal to 0.05 were considered to be statistically significant.

\section{Results}

In total, 57 patients were included in the study; of these, 29 (50.88\%) were male and 28 (49.12\%) were female patients, with a median age of 55.9 years (range, 24-90 years). Of the 57 patients, 9 were presented with recurrent asthma symptoms. The baseline characteristics of these patients with regards to clinical features are shown in Table 1. With Cough (57/57), expectoration (57/57) and wheezing (57/57) were the most common symptoms. Less common symptoms were chest tightness (35/57), fever (25/57), fatigue (21/57), haemoptysis (13/57) and headache (6/57). The clinical stages of these 57 patients are shown in Table 1 .

For the 57 patients, the time of first hospital visit to final 
Table 1 The clinical features, stages, Ig E test and radiologic manifestation of the $57 \mathrm{ABPM}$ patients

\begin{tabular}{|c|c|c|}
\hline Characteristics & No. of patients $(n)$ & Percentage (\%) \\
\hline \multicolumn{3}{|l|}{ Symptoms/signs } \\
\hline Cough & $57 / 57$ & 100.0 \\
\hline Expectoration & $57 / 57$ & 100.0 \\
\hline Wheezing & $57 / 57$ & 100.0 \\
\hline Chest tightness & $35 / 57$ & 61.4 \\
\hline Fever & $25 / 57$ & 43.9 \\
\hline Fatigue & $21 / 57$ & 36.8 \\
\hline Haemoptysis & $13 / 57$ & 22.8 \\
\hline Headache & $6 / 57$ & 10.5 \\
\hline wheezes & $37 / 57$ & 64.9 \\
\hline crackles & $28 / 57$ & 49.1 \\
\hline \multicolumn{3}{|l|}{ Clinical stage } \\
\hline $\begin{array}{l}\text { Glucocorticoid dependent } \\
\text { asthma [5] }\end{array}$ & $26 / 57$ & 45.6 \\
\hline Exacerbation [3] & $18 / 57$ & 31.6 \\
\hline Remission [4] & $9 / 57$ & 15.8 \\
\hline advanced [6] & $2 / 57$ & 3.5 \\
\hline Acute [1] & $2 / 57$ & 3.5 \\
\hline \multicolumn{3}{|l|}{ Specific lg E test } \\
\hline A. fumigatus & $39 / 57$ & 68.4 \\
\hline Polyvalent fungus & $26 / 57$ & 45.6 \\
\hline Candida albicans & $20 / 57$ & 35.1 \\
\hline $\begin{array}{l}\text { A. fumigatus + Polyvalent } \\
\text { A. fumigatus + Candida } \\
\text { albicans }\end{array}$ & $15 / 57$ & 26.3 \\
\hline $\begin{array}{l}\text { A. fumigatus + Polyvalent } \\
\text { fungus }\end{array}$ & $7 / 57$ & 12.3 \\
\hline $\begin{array}{l}\text { A. fumigatus + Candida } \\
\text { albicans }\end{array}$ & $2 / 57$ & 3.5 \\
\hline \multicolumn{3}{|l|}{ Radiological manifestations } \\
\hline Central bronchiectasis & $33 / 57$ & 57.9 \\
\hline Mucus plug & $16 / 57$ & 28.1 \\
\hline Mediastinal adenopathy & $14 / 57$ & 24.6 \\
\hline Patch and stripe shadows & $14 / 57$ & 24.6 \\
\hline Aspergilloma & $10 / 57$ & 17.5 \\
\hline Fibrosis & $7 / 57$ & 12.3 \\
\hline Pulmonary bulla & $2 / 57$ & 3.5 \\
\hline Consolidation & $1 / 57$ & 1.8 \\
\hline
\end{tabular}

ABPM, allergic bronchopulmonary mycosis.
Table 2 Misdiagnoses of the ABPM patients

\begin{tabular}{lcc}
\hline Misdiagnosed disease & No. of patients $(\mathrm{n})$ & Percentage (\%) \\
\hline COPD & $10 / 22$ & 45.5 \\
Bronchiectasis & $6 / 22$ & 27.3 \\
Pneumonia & $3 / 22$ & 13.6 \\
DPB & $1 / 22$ & 4.5 \\
Lung cancer & $1 / 22$ & 4.5 \\
Tuberculosis & $1 / 22$ & 4.5 \\
\hline ABPM, allergic bronchopulmonary mycosis; COPD, chronic \\
obstructive pulmonary disease; DPB, diffuse panbronchiolitis.
\end{tabular}

diagnosis ranged from as short as 24 days to a period of 40 years (average $137.37 \pm 117.39$ months). A majority of cases $(22 / 57)$ were misdiagnosed or underdiagnosed as seen in Table 2. Not including asthma as a stand-alone diagnosis in 9 patients, the other patients in the top 3 included chronic obstructive pulmonary disease (COPD), bronchiectasis and pneumonia in 10, 6 and 3 cases respectively. Only 26 $(45.6 \%)$ were correctly diagnosed without delay.

Out of 57 patients, eosinophilia $\left(\geq 1,000 / \mathrm{mm}^{3}\right)$ was detected in 11 patients (19.3\%). An elevated total serum Ig E level $(\geq 1,000 \mathrm{kU} / \mathrm{L})$ was detected in 25 of the 57 patients (43.9\%). All of the patients were tested for specific Ig E (sp-Ig E), in which 39 were only Aspergillus fumigatus spIg $\mathrm{E}^{+}(68.4 \%), 26$ were only polyvalent fungus (Penicillium notatum, Cladosporium, Aspergillus fumigatus, Candida albicans, Alternaria alternata, Helminthosporium carbonum) sp-Ig E ${ }^{+}$ (45.6\%), 20 were only Candida albicans sp-Ig E $\mathrm{E}^{+}(35.1 \%), 15$ were Aspergillus fumigatus, polyvalent fungus and Candida albicans sp-Ig E $\mathrm{E}^{+}(26.3 \%), 7$ were both Aspergillus fumigatus and Polyvalent fungus sp- $\operatorname{Ig~E} \mathrm{E}^{+}(12.3 \%), 2$ were both Aspergillus fumigatus, and Candida albicans $\mathrm{sp}-\mathrm{Ig} \mathrm{E} \mathrm{E}^{+}(3.5 \%)$ (Table 1). In addition, 40 patients were positive by sputum microscopy and culture. Sputum was positive for Aspergillus species in 31.6\% (18 of 57 cases), while positive for Candida species in $38.6 \%$ (22 of 57 cases) (Table 3).

Various chest CT abnormalities were noted in our patients as indicated in Table 1. Central bronchiectasis was seen in 33 patients. As might be expected, central bronchiectasis $(57.9 \%)$ and mucus plug $(28.1 \%)$ were the most common manifestations. Aspergillomas, which moved to dependent positions on prone scans, were noted in dilated bronchiectatic cavities in 10 patients $(17.5 \%)$.

Lung function was also tested in 34 patients. A mild to moderate obstructive pattern was observed in 32 cases. 
Table 3 The sputum cultures for fungus of the 57 ABPM patients

\begin{tabular}{lcc}
\hline Sputum cultures for fungus & No. of patients (n) & Percentage (\%) \\
\hline Candida & $22 / 57$ & 38.6 \\
Candida albicans & $19 / 57$ & 33.3 \\
Krusei & $2 / 57$ & 3.5 \\
Candida glabrata & $1 / 57$ & 1.8 \\
Aspergillus & $18 / 57$ & 31.6 \\
Aspergillus fumigatus & $12 / 57$ & 21.1 \\
Aspergillus flavus & $2 / 57$ & 3.5 \\
Aspergillus terreus & $2 / 57$ & 3.5 \\
Aspergillus niger & $1 / 57$ & 1.8 \\
Aspergillus versicolor & $1 / 57$ & 1.8 \\
\hline
\end{tabular}

ABPM, allergic bronchopulmonary mycosis.

Additionally, bronchofiberscopy was performed in 14 patients. By bronchofiberscopic observation, bronchial lumen was seen to have significant inflammation in 12 patients, corresponding to an eosinophilic infiltration to the bronchial walls in pathologic findings of bronchial biopsy specimens. The mean number of cells per bronchoalveolar lavage fluid (BALF) in 6 patients was approximately 1.8 to $5\left(\times 10^{6}\right)$. The predominant cell type was neutrophil (65-90\%), followed by macrophage (10$20 \%)$, lymphocyte (3-9\%) and eosinophil (2-7\%).

After 3 weeks of treatment, clinical and radiological improvement was observed in all the patients. The total Ig E level at baseline was 1,148.0 KU.L-1, after treatment with glucocorticoid, itraconazole or glucocorticoid and itraconazole, the mean absolute decline in total $\mathrm{Ig} \mathrm{E}$ value was 316,325 and 316 respectively (Table 4). On the other hand, the trend of the number of total eosinophil count cells was inconsistent. The mean number of total eosinophil count cells at baseline was $0.215 \mu \mathrm{L}^{-1}$, and after treatment with glucocorticoid, itraconazole or glucocorticoid and itraconazole, the mean number of total eosinophil count cells was $0.20,1.06$ and 0.125 respectively (Table 4 ).

\section{Discussion}

$\mathrm{ABPM}$ is an allergic pulmonary disease caused by a hyperimmune response to the certain environmental fungi. It was first reported in 1952 by Hinson et al., which was caused by Aspergillus fumigatus (4). Since then, hundreds of patients with this disorder were reported, who were caused by A. flavus, A. niger, Candida albicans, Penicillium or Schizophylum commune, other than Aspergillus fumigatus (20,21). However, several patients who presented to the hospital were diagnosed delay as long as more than 10 years (22). In our present study, 1 patient was believed to be undiagnosed for 40 years. Thus, the most troubling aspect in dealing with $A B P M$ may be related to early recognition of this disorder.

There may be many reasons for the underdiagnosis of ABPM. Firstly, ABPM is usually described in patients with pre-existing asthma or cystic fibrosis, however, it is not absolutely essential (23). In the current study, preexisting asthma was only present in 9 patients and 48 patients were nonasthmatic, while preexisting cystic fibrosis was not present in total patients. Thus, ABPM is not a common disease even rare outside of the diagnosis of asthma or cystic fibrosis, which could easily go unrecognized.

Secondly, it is well-known that patients with ABPA were classified in to 7 stages (24). A previous study showed that peripheral blood eosinophilia is usually not present and pulmonary infiltrates are absent in the remission stage. Moreover, precipitating antibodies against fungus species may not be present in stages other than acute stage (23). Therefore, the patients in some stages of ABPM could be missed, who did not satisfy the clinical criteria due to the absence of eosinophilia and/or the technical limitations of immunological investigations (24).

Thirdly, some practitioners may not recognize the signs or symptoms of this disease, as a result of misdiagnosis. In our present study, nearly a half of cases $(22 / 57)$ were misdiagnosed, while only $35(61.4 \%)$ were correctly diagnosed without delay. A similar observation was recorded by other authors; a majority of cases (44/70) were misdiagnosed, while Only 18 (26\%) were correctly diagnosed as ABPM (22). Thus, a deeper awareness or understanding of ABPM should be heightened and paid more attention in physicians.

One of the important findings in the present report was that 10 patients had been treated with bronchodilator drugs before coming to this hospital as they had been diagnosed as cases of COPD. Few patients with ABPM have been reported in clinical conditions such as COPD, other than asthma or cystic fibrosis. To date, only 3 reports documented the presence of ABPM concomitant with COPD in patients (25-27). Agarwal et al. reported the first report of such an association, who presented with an exacerbation of COPD due to co-existent ABPM (26). There is a pathophysiological plausibility of a link between 
Table 4 The total-Ig E level of the ABPM patients before and after treatment

\begin{tabular}{|c|c|c|c|c|}
\hline Immunological findings & $\begin{array}{l}\text { Before treatment } \\
\qquad(\mathrm{n}=57)\end{array}$ & \multicolumn{3}{|c|}{ After 3 months treatment $(n=48)$} \\
\hline Total Ig E levels $\mathrm{KU} \cdot \mathrm{L}^{-1}$ & $1,148.0(10.5-5,920)$ & $316.0(78.6-4,371)^{\star}$ & $325.0(10-5,850)^{*}$ & $316.0(25-5,000)^{*}$ \\
\hline Total eosinophil count cells $\mu \mathrm{L}^{-1}$ & $0.215(0-17.8)$ & $0.20(0-0.64)$ & $1.06(0.01-1.32)$ & $0.125(0-14.3)$ \\
\hline
\end{tabular}

All the values are expressed as mean (95\% confidence intervals). *, P value $\leq 0.05$ statistically significant after 3 months treatment compared to before treatment. ABPM, allergic bronchopulmonary mycosis.

$\mathrm{ABPM}$ and COPD, as the patients with COPD have mucus hypersecretion and impaired mucociliary clearance, where fungus could be trapped in and then germinate to form hyphae (28). Thus, COPD may be a predisposing factor for $\mathrm{ABPM}$, which could develop the co-existence of COPD and ABPM. Therefore, co-existent ABPM should be considered in patients with exacerbation of COPD who are unresponsive to routine therapies, and these patients should do further examination as soon as possible.

The most important changes of HRCT chest in our present study showed central bronchiectasis and mucus plug, which is considered as highly suggestive of ABPM $(29,30)$. Spirometry showed mild to moderate obstruction mostly, which may be due to bronchiectasis at presentation in most patients in the present study. The lung function test could not reveal any specific pattern to help in the diagnosis of ABPM, but could be used in the assessment of the severity of impairment of lung function (29). With help from radiographic findings, 14 patients also underwent bronchoscopy and showed similar results, providing support that discharge of mucus plugs is not a valid diagnostic criterion for $\mathrm{ABPM}(31,32)$ but could relieve uncomfortable symptoms such as wheezing and chest tightness.

Aspergillus fumigatus is regarded to be the most common organism implicated in the causation of ABPM, however, Candida albicans was also commonly isolated in the present series. The positive for Candida albicans species in sputum was observed in $33.3 \%$ of cases as compared to positive for Aspergillus fumigatus which were found in 31.6\% cases. In addition, the positive for Candida albicans sp-Ig $\mathrm{E}$ was measured in $35.1 \%$ of cases, while the positive for Aspergillus fumigatus sp-Ig E was detected in $68.4 \%$ cases. In the study, the culture was made with bronchoscopic gained material or sputum. All sputum samples were collected with standard method and quality control to avoid the oral contamination. The diagnosis of candida-ABPM was made based on results of BALF or at least three-times positive sputum culture. Therefore, Candida albicans is also frequent causative mycoses of ABPM, which is present greatly in the mouth of normal people. Before visiting our hospital, most patients have sought medical service for a long time and many kinds of antibiotics have been used, which inhibit normal bacteria flora and cause flora imbalance. This maybe one reason for high frequency of Candida in this study.

Furthermore, we also detected the level of total Ig E and the number of total eosinophil count cells during followup and found that a total Ig E value significantly decreased while eosinophilia in the peripheral blood did not change regularly after 3 weeks of treatment. Therefore, the serum total $\mathrm{Ig} \mathrm{E}$ is a useful investigation either in the initial diagnostic workup or during follow-up, while the total peripheral blood eosinophil count is not a good screening test for ABPM (29).

\section{Conclusions}

In conclusion, ABPM due to fungi other than aspergilli is not common and currently underdiagnosed, thus, comprehensive basic and clinical studies are needed to establish more elaborate diagnostic criteria for patients with ABPM. Increased awareness among physicians is also required to prevent misdiagnosis of cases, especially in those patients with exacerbation of COPD. Furthermore, once the diagnosis has been confirmed, treatment should be initiated, and ongoing follow-up continued with detecting total Ig E level throughout the course of this chronic lung disorder.

\section{Acknowledgments}

Funding: This work was supported by Beijing Science and Technology Committee Project, Capital Public Health Incubation Program (Z141100002114006), Capital Health Development Research Project, Independent Innovation 
Program (CD2014-2-1062), the National Nature Science Foundation of China (L1422025) and National Key R\&D Program of China (2016YFC0206502).

\section{Footnote}

Conflicts of Interest: The authors have no conflicts of interest to declare.

Ethical Statement: The authors are accountable for all aspects of the work in ensuring that questions related to the accuracy or integrity of any part of the work are appropriately investigated and resolved. This is a retrospective study and ethical approval is not required.

\section{References}

1. Ishiguro T, Takayanagi N, Uozumi R, et al. Diagnostic criteria that can most accurately differentiate allergic bronchopulmonary mycosis from other eosinophilic lung diseases: A retrospective, single-center study. Respir Investig 2016;54:264-71.

2. Knutsen AP, Bush RK, Demain JG, et al. Fungi and allergic lower respiratory tract diseases. J Allergy Clin Immunol 2012;129:280-91; quiz 292-3.

3. Oshikata C, Watanabe M, Saito A, et al. Allergic Bronchopulmonary Mycosis due to Exposure to Eurotium herbariorum after the Great East Japan Earthquake. Prehosp Disaster Med 2017;32:688-90.

4. Hinson KF, Moon AJ, Plummer NS. Broncho-pulmonary aspergillosis; a review and a report of eight new cases. Thorax 1952;7:317-33.

5. Sandhu RS, Mehta SK, Khan ZU, et al. Role of Aspergillus and Candida species in allergic bronchopulmonary mycoses. A comparative study. Scand J Respir Dis 1979;60:235-42.

6. Shah A, Maurya V, Panjabi C, et al. Allergic bronchopulmonary aspergillosis without clinical asthma caused by Aspergillus niger. Allergy 2004;59:236-7.

7. Dyer ZA, Wright RS, Rong IH, et al. Back pain associated with endobronchial mucus impaction due to Bipolaris australiensis colonization representing atypical Allergic Bronchopulmonary Mycosis. Med Mycol 2008;46:589-94.

8. González de Olano D, González-Mancebo E, Gandolfo Cano M, et al. Successful treatment of allergic bronchopulmonary candidiasis with a recombinant antiimmunoglobulin E antibody. J Investig Allergol Clin
Immunol 2009;19:416-7.

9. McAleer R, Kroenert DB, Elder JL, et al. Allergic bronchopulmonary disease caused by Curvularia lunata and Drechslera hawaiiensis. Thorax 1981;36:338-44.

10. Toyotome T, Satoh M, Yahiro M, et al. Glucoamylase is a major allergen of Schizophyllum commune. Clin Exp Allergy 2014;44:450-7.

11. Chowdhary A, Agarwal K, Kathuria S, et al. Allergic bronchopulmonary mycosis due to fungi other than Aspergillus: a global overview. Crit Rev Microbiol 2014;40:30-48.

12. Bhankhur D, Singla N, Aggarwal D, et al. Prevalence of allergic bronchopulmonary aspergillosis among patients with severe bronchial asthma in a tertiary care hospital in Northern India. Indian J Pathol Microbiol 2019;62:111-3.

13. Dhooria S, Agarwal R. Diagnosis of allergic bronchopulmonary aspergillosis: a case-based approach. Future Microbiol 2014;9:1195-208.

14. Greenberger PA. Allergic bronchopulmonary aspergillosis. J Allergy Clin Immunol 2002;110:685-92.

15. Kirsten D, Nowak D, Rabe KF, et al. [Diagnosis of bronchopulmonary aspergillosis is often made too late]. Med Klin (Munich) 1993;88:353-6.

16. Kim Y, Lee HY, Gu KM, et al. Delayed diagnosis of allergic bronchopulmonary aspergillosis due to absence of asthmatic symptoms. Asia Pac Allergy 2016;6:187-91.

17. Rosenberg M, Patterson R, Mintzer R, et al. Clinical and immunologic criteria for the diagnosis of allergic bronchopulmonary aspergillosis. Ann Intern Med 1977;86:405-14.

18. Agarwal R, Khan A, Garg M, et al. Pictorial essay: Allergic bronchopulmonary aspergillosis. Indian J Radiol Imaging 2011;21:242-52.

19. Aggarwal AN, Gupta D, Jindal SK. Development of a simple computer program for spirometry interpretation. J Assoc Physicians India 2002;50:567-70.

20. Matsuse H, Nakata H, Fukahori S, et al. A clinical evaluation of definitive and clinical allergic bronchopulmonary mycosis. Intern Med 2006;45:759-62.

21. Deepak D, Singh Rajput M, Sharma B, et al. Allergic Bronchopulmonary Mycosis due to fungi other than Aspergillus. Eur Ann Allergy Clin Immunol 2019;51:75-9.

22. Mou Y, Ye L, Ye M, et al. A retrospective study of patients with a delayed diagnosis of allergic bronchopulmonary aspergillosis/allergic bronchopulmonary mycosis. Allergy Asthma Proc 2014;35:e21-6.

23. Chakrabarti A, Sethi S, Raman DS, et al. Eight-year study 
of allergic bronchopulmonary aspergillosis in an Indian teaching hospital. Mycoses 2002;45:295-9.

24. Ishiguro T, Takayanagi N, Kagiyama N, et al. Clinical characteristics of biopsy-proven allergic bronchopulmonary mycosis: variety in causative fungi and laboratory findings. Intern Med 2014;53:1407-11.

25. Agarwal R, Hazarika B, Gupta D, et al. Aspergillus hypersensitivity in patients with chronic obstructive pulmonary disease: COPD as a risk factor for ABPA? Med Mycol 2010;48:988-94.

26. Agarwal R, Srinivas R, Jindal SK. Allergic bronchopulmonary aspergillosis complicating chronic obstructive pulmonary disease. Mycoses 2008;51:83-5.

27. Mir E, Shah A. Allergic bronchopulmonary aspergillosis in a patient with chronic obstructive pulmonary disease. Prim Care Respir J 2012;21:111-4.

Cite this article as: Feng H, Lv P, Ren X, Dai H, Yang T. Misinterpretation of allergic bronchopulmonary aspergillosis/ allergic bronchopulmonary mycosis due to diverse characteristics in different clinical stages. J Thorac Dis 2019;11(11):4484-4491. doi: 10.21037/jtd.2019.10.78
28. Agarwal R. Allergic bronchopulmonary aspergillosis. Chest 2009;135:805-26.

29. Agarwal R, Sehgal IS, Dhooria S, et al. Developments in the diagnosis and treatment of allergic bronchopulmonary aspergillosis. Expert Rev Respir Med 2016;10:1317-34.

30. Kaur M, Sudan DS. Allergic Bronchopulmonary Aspergillosis (ABPA)-The High Resolution Computed Tomography (HRCT) Chest Imaging Scenario. J Clin Diagn Res 2014;8:RC05-7.

31. Khalil KF. Therapeutic bronchoalveolar lavage with conventional treatment in allergic bronchopulmonary aspergillosis. J Coll Physicians Surg Pak 2015;25:359-62.

32. Tamura A, Hebisawa A, Kurashima A, et al. The use of bronchofiberscopy for diagnosis of allergic bronchopulmonary aspergillosis. Intern Med 1997;36:865-9. 